\title{
Decomposição de resíduos vegetais em ambiente alagado e sequeiro sob diferentes manejos de solo
}

\author{
Decomposition of plant residues in a wet and dry environment under different soil \\ management \\ L. Neto ${ }^{1}$; C. Coradini ${ }^{2}$; E. Junges ${ }^{3 *} ;$ C. Michelon ${ }^{3}$ \\ ${ }^{l}$ Doutoranda em Ciência do Solo, Departamento de Solos, Universidade Federal de Santa Maria, CEP 97105 - \\ 900, Santa Maria-RS, Brasil \\ ${ }^{2}$ Doutor em Agronomia, Universidade Federal de Santa Maria, CEP 97105-900, Santa Maria-RS, Brasil \\ ${ }^{3}$ Professores do Departamento de Agricultura, Instituto Federal Farroupilha, CEP 97420-000, São Vicente-RS, \\ Brasil \\ *emanuele.junges@iffarroupilha.edu.br
}

(Recebido em 22 de abril de 2017; aceito em 18 de dezembro de 2017)

\begin{abstract}
Os resíduos culturais são os principais substratos para os estoques de matéria orgânica do solo, sendo formados basicamente pelos mesmos componentes: nitrogênio, fração solúvel, concentrações de celulose + hemicelulose, lignina. Porém, em diferentes proporções as quais definem seu grau de qualidade. Assim, a qualidade do resíduo e o tipo de manejo estão entre os principais fatores que controlam as taxas de decomposição. Neste sentido, o objetivo deste estudo foi avaliar a decomposição de resíduos de nabo forrageiro (Raphanus sativus L.) e azevém (Lolium multiflorum), manejados sob sistema plantio direto e convencional em um Argissolo em ambiente de sequeiro e um Planossolo em área alagada. $\mathrm{O}$ estudo foi conduzido durante 90 dias na área experimental do Instituto Federal Farroupilha, Campus São Vicente do Sul, utilizando resíduos de nabo forrageiro e de azevém em um Argissolo Vermelho Distrófico arênico e um Planossolo Háplico Eutrófico arênico. Os resíduos foram avaliados na superfície e incorporados ao solo. Aos 30, 60 e 90 dias foram avaliados a porcentagem de decomposição. A incorporação dos resíduos acelerou o processo de decomposição, onde esse processo foi mais acentuado a partir de 60 dias. Independente do manejo adotado, os resíduos de nabo forrageiro foram decompostos mais rapidamente do que os de azevém. O tipo de solo afetou o processo de decomposição, no Argissolo obteve os maiores valores de decomposição em relação ao Planossolo.

Palavras-chave: Mineralização, Raphanus sativus, Lolium multiflorum
\end{abstract}

Cultural residues are the main substrates for soil organic matter stocks, being formed basically by the same components. However, in different proportions which define their degree of quality. Thus, the quality of the waste and the type of management are among the main factors controlling the rates of decomposition. In this sense, the objective of this study was to evaluate the decomposition of Raphanus sativus and Lolium multiflorum residues, managed under no-tillage and conventional system in an Ultisol in a rainfed environment and a Planosol in a flooded area. The study was conducted during 90 days in the experimental area of the Federal Institute Farroupilha, Campus São Vicente do Sul, using plant residues of Raphanus sativus and Lolium multiflorum in an Argisol and Planosol. The residues were evaluated on the surface and incorporated into the soil. At 30,60 and 90 days the percentage of decomposition was evaluated. The incorporation of the residues accelerated the decomposition process from 60 days. Regardless of the management adopted, $R$. sativus residues were decomposed faster than $L$. multiflorum. The soil type affected the decomposition process, in the Ultisol obtained the highest decomposition values in relation to the Planosol.

Keywords: Mineralization, Raphanus sativus, Lolium multiflorum

\section{INTRODUÇÃO}

Os solos agrícolas têm uma importante função na mitigação de mudanças climáticas [26], uma vez que o manejo pode aumentar ou diminuir os estoques de matéria orgânica do solo, além de alterar as emissões de gases do efeito estufa. Os resíduos culturais são a principal fonte de matéria orgânica para o solo e a sua decomposição é considerada um processo variável. Esse processo é 
influenciado pelas características físicas e químicas do solo, da composição química e da localização desses resíduos [28].

Os resíduos culturais são compostos basicamente dos mesmos componentes, como por exemplo, compostos solúveis, lignina, porém em diferentes proporções [11]. De acordo com as proporções desses componentes, os resíduos podem ser classificados de alta ou baixa qualidade. Os resíduos de baixa relação $\mathrm{C}: \mathrm{N}$ e ricos em fração solúvel são classificados como de alta qualidade, sendo rapidamente decompostos [22], como exemplo se destacam as fabaceas e crucíferas. Os resíduos culturais de baixa qualidade são caracterizados por possuírem uma alta relação C:N e pobres em fração solúvel sendo decompostos mais lentamente [5], a exemplo das Poaceas.

A utilização de Fabaceas e crucíferas da família Brassicaceae como plantas de cobertura constitui uma importante fonte de nitrogênio ao solo. Apesar dessas características, seu uso no Brasil ainda é pequeno em relação às gramíneas como azevém (Lolium multiflorum L.). Isso ocorre pelo maior custo de produção, baixo rendimento de matéria seca, desenvolvimento inicial lento e rápida decomposição [9]. Por outro lado, quando é feita a utilização de poaceas no outono/inverno, podem ocorrer prejuízos as culturas subsequentes em função da provável imobilização de nutrientes como o $\mathrm{N}$ durante a sua decomposição [27, 15]. Neste sentido, o conhecimento da taxa de decomposição dos diferentes resíduos e consequente liberação de nutrientes são fundamentais para potencializar a ciclagem e o aproveitamento de nutrientes pelas próximas culturas, desta forma, reduzindo os impactos ambientais das práticas agrícolas [3, 13].

Além disso, a localização dos resíduos aliada ao preparo do solo exerce uma grande influência na velocidade de decomposição dos mesmos. Os resíduos mantidos em superfície são decompostos lentamente em relação aos incorporados [6], pois a decomposição é promovida pelas alterações microclimáticas relacionadas ao contato do resíduo com o solo [21]. O tipo de solo é outro fator de grande influência nesses processos. Em solos de drenagem restrita, a decomposição dos resíduos usualmente ocorre em menores taxas do que em solos aerados [28].

Estudos avaliando a decomposição de diferentes resíduos sob diferentes manejos estão associadas a sistemas de sequeiro. Portanto, surge a necessidade de avaliar o processo de decomposição de resíduos contrastantes em ambientes anaeróbios e de sequeiros. Neste sentido, o objetivo deste estudo foi avaliar a decomposição de resíduos de nabo forrageiro (Raphanus sativus L.) e azevém (Lolium multiflorum), manejados sob sistema plantio direto e convencional em um Argissolo em ambiente de sequeiro e um Planossolo em área alagada.

\section{MATERIAL E MÉTODOS}

O estudo foi conduzido no ano de 2016 na área experimental do Instituto Federal Farroupilha (IFFar), Campus São Vicente do Sul, (Latitude 29 41' 30' ' S, Longitude 54 40' 46' ' W, altitude de 129 metros). A área está localizada na região centro-oeste do Rio Grande do Sul. O clima é classificado como subtropical úmido, tipo "Cfa" segundo a classificação de Köppen, com precipitação média anual de $1445 \mathrm{~mm}$.

\section{Solos}

O experimento foi implantado em dois tipos de solo: 1) Argissolo Vermelho Distrófico arênico, em condição de sequeiro. 2) Planossolo Háplico Eutrófico arênico, em ambiente de várzea. $\mathrm{O}$ solo 1 possui as seguintes características químicas: $\mathrm{pH} 5,5 ; \mathrm{Ca} 5,9 \mathrm{cmolc} \mathrm{dm}^{-3} ; \mathrm{Mg} 1,9$ cmolc dm ${ }^{-3}$; M.O. 3\%; P 25,6 mg dm${ }^{-3} ; \mathrm{K} 68 \mathrm{mg} \mathrm{dm}^{-3} \mathrm{e}$, as características químicas do solo 2 são: pH 6,2; Ca 11,8 cmolc dm${ }^{-3}$; Mg 1,6 cmolc dm ${ }^{-3}$; M.O. 3\%; P 27,5 mg dm${ }^{-3} ; \mathrm{K} 100 \mathrm{mg} \mathrm{dm}^{-3}$.

\section{Resíduos culturais utilizados}

Foram utilizados resíduos culturais de nabo forrageiro (Raphanus sativus) e de azevém (Lolium multiflorum). A coleta dos resíduos vegetais foi realizada quando as culturas se 
encontravam no estágio de maturação fisiológica. A parte aérea foi obtida cortando-se as plantas rente ao solo, e o material triturado com tesoura em pedaços de aproximadamente $3 \mathrm{~cm}$.

\section{Desenho experimental}

Os tratamentos consistiram na combinação de dois solos, dois tipos de resíduos culturais e dois sistemas de manejo (resíduos incorporados e em superfície) resultando em oito tratamentos: T1Argissolo + palha de nabo em superfície; T2- Planossolo + palha de nabo em superfície; T3Argissolo + palha de nabo incorporada; T4- Planossolo + palha de nabo incorporada; T5Argissolo + palha de azevém em superfície; T6- Planossolo + palha de azevém em superfície; T7Argissolo + palha de azevém incorporada; T8- Planossolo + palha de azevém incorporada.

\section{Avaliações}

Para avaliação da decomposição da matéria seca foram utilizados litterbags. Pesou-se 50 gramas de resíduos das culturas que foram picados e colocados em sacos de nylon com dimensões de 10 x $15 \mathrm{~cm}$ e malha $2 \mathrm{~mm}$. Posteriormente, os litterbags foram distribuídos aleatoriamente no campo, com uma distância de aproximadamente 1 metro na superfície do solo para os tratamentos com resíduos em superfície, e outro conjunto incorporado ao solo a uma profundidade de $5 \mathrm{~cm}$ para os tratamentos com resíduos incorporados.

Em cada data de coleta (30, 60 e 90 dias após a implantação), três repetições eram retiradas de cada tratamento, os litterbags passaram por uma pré-limpeza para a retirada de solo aderido aos resíduos. Após esse processo, as amostras foram secas em estufa de circulação de ar forçada a $60^{\circ} \mathrm{C}$ até atingirem peso constante. Posteriormente foi realizada uma nova pesagem para determinação da massa seca.

\section{Análise estatística}

Foi avaliada a decomposição de resíduos de nabo forrageiro e azevém, em superfície ou incorporados em dois tipos de solo em três períodos de coleta: 30, 60 e 90 dias. Os tratamentos foram arranjados em esquema trifatorial 2 X 2 X 2 (resíduos culturais X preparo do solo X tipo de solo).

Os dados foram submetidos a análise de variância, sendo testada a interação entre os fatores. As médias dos tratamentos foram comparadas utilizando-se o teste de Tukey a 5\%. Todos os dados foram analisados usando o programa estatístico SISVAR.

\section{RESULTADOS E DISCUSSÃO}

A dinâmica de decomposição é normalmente caracterizada por apresentar duas fases distintas: a primeira mais rápida e intensa, seguida de uma fase mais lenta $[4,6]$. Neste estudo, a decomposição dos resíduos apresentou comportamento semelhante durante os períodos de avaliação. Na primeira, 30 dias após a adição dos resíduos ao solo, houve uma rápida decomposição dos resíduos de nabo em relação aos de azevém (Figura 1), não havendo diferença significativa entre os manejos adotados. Tal comportamento está associado a mineralização dos compostos carbonados mais lábeis como os açúcares $[25,7]$ presentes nos resíduos de baixa relação C:N. Após a exaustão das frações lábeis, a decomposição torna-se gradativa em taxas mais lentas, pois ocorre a permanência de compostos mais resistentes a atividade microbiana, como compostos lignocelulóticos [2,1]. Este estudo sugere que até os 90 dias as frações lábeis possivelmente não haviam sido esgotadas.

Os resultados observados aos 30 dias, onde estão relacionados ao tipo de solo também obtiveram efeito significativo, sendo os maiores valores de decomposição encontrados no Argissolo em relação ao Planossolo (Figura 1). Estando de acordo com Finn et al. (2015) [10], os autores relatam uma maior degradação dos resíduos em solos com maior teor de argila do que em solos de fração mais grossa, como os arenosos. Cotrufo et al. (2015) [8], verificaram que a capacidade de retenção e estabilização do carbono no solo é maior em solos de textura fina, ricos 
em óxidos e filossilicatos os quais são relacionados aos teores de argila. Além disso, o ambiente alagado do Planossolo interferiu neste processo.

$\longrightarrow$ —- Resíduos de Nabo Forrageiro
$\longrightarrow$ - Resíduo de Azevém
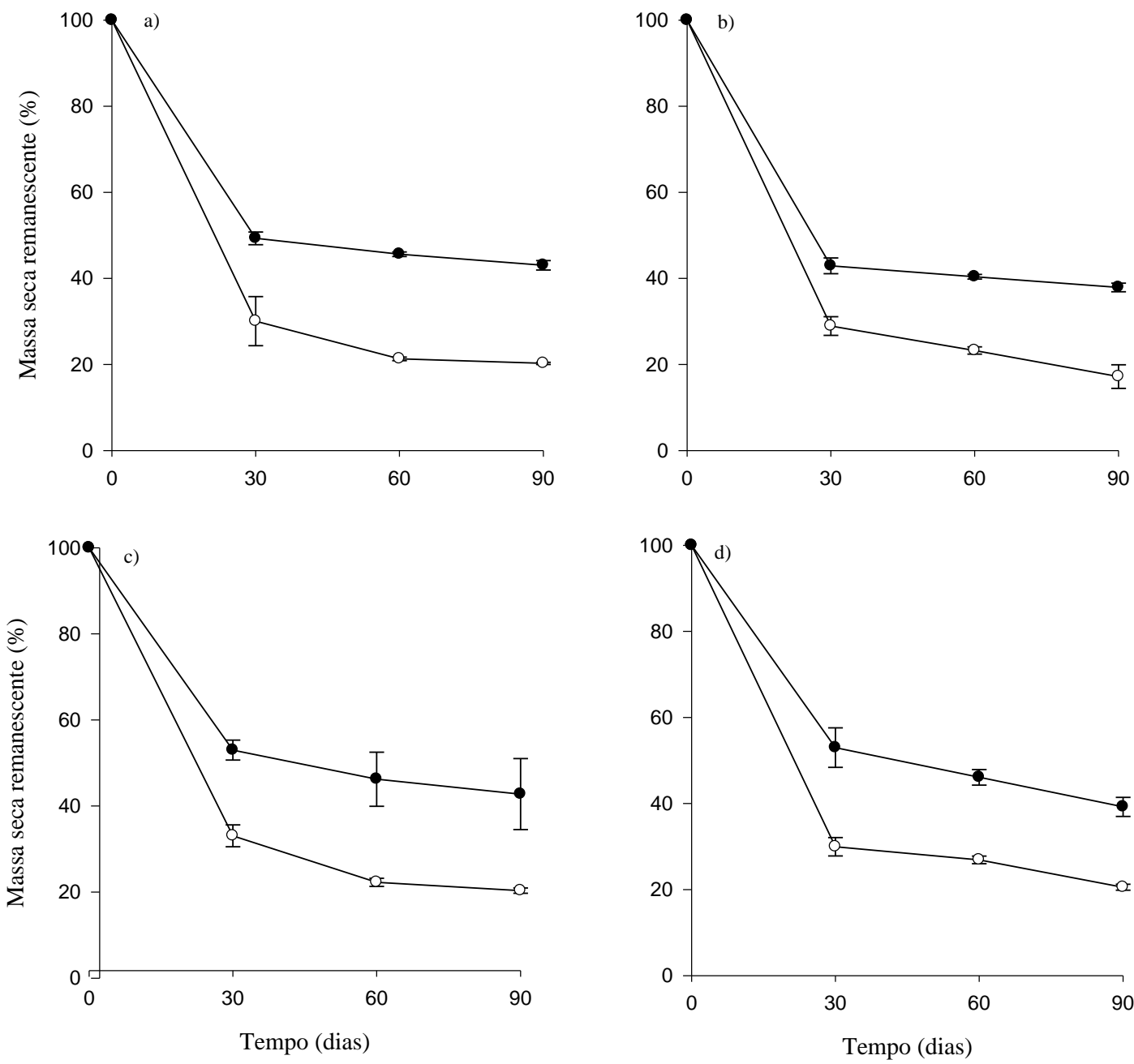

Figura 1: a) Resíduos de nabo forrageiro (Raphanus sativus L.) e de azevém (Lolium multiflorum L.) mantidos em superfície em um Argissolo; b) Resíduos de nabo forrageiro e azevém incorporados em um

Argissolo; c) Resíduos de nabo forrageiro e azevém mantidos em superfície em um Planossolo; $d$ ) Resíduos de nabo forrageiro e azevém incorporados em um Planossolo.

Além da textura do solo, outro fator que deve ser considerado é o sistema de manejo em cada solo. Em solos de drenagem restrita, como os Planossolos, a decomposição na ausência de oxigênio ocorre em taxas menores do que o mesmo processo em solos aerados, sendo dependente de receptores de elétrons como íons de ferro, manganês e sulfato [19,23]. De acordo com Olk et al. (2002) [20], a baixa disponibilidade de oxigênio nos solos de várzea propicia a lenta degradação, principalmente de compostos recalcitrantes, como a lignina, o que contribui para uma maior estabilização de compostos fenólicos no solo.

Aos 60 dias de avaliação, a dinâmica da decomposição seguiu o mesmo comportamento apresentado no primeiro período de avaliação. No entanto, houve a interação entre o resíduo e o manejo adotado (Figura 1). Novamente os resíduos de nabo apresentaram maiores taxas de decomposição em relação aos de azevém. Esses valores foram mais elevados quando estes resíduos foram incorporados ao solo. Resultados semelhantes foram encontrados por Coppens et 
al. (2006) com resíduos de colza (Brassica napus L.) e Mazzocini et al. (2016) [18] com resíduos de arroz (Oryza sativa), quando avaliaram a influência do manejo na decomposição dos mesmos.

A decomposição de resíduos incorporados ao solo é favorecida pelo ambiente, pois há maior contato do resíduo com o solo, o que favorece a disponibilidade de água e nutrientes junto ao sítio de decomposição [17]. A maior decomposição dos resíduos de nabo quando comparado aos resíduos de azevém, se deve as características químicas do resíduo de nabo. De acordo com Recous et al. (1995) [24], a disponibilidade de nitrogênio nos resíduos possui um importante papel nos processos de decomposição, pois é um fator limitante no desenvolvimento microbiano.

Ao final do período de avaliação os resíduos ainda apresentavam crescentes valores de decomposição, com destaque para os resíduos de nabo forrageiro. Neste período, além dos resíduos, houve o efeito isolado do manejo, sendo que os resíduos incorporados foram os que obtiveram as maiores taxas de decomposição.

Tabela 1: Resultado da análise de variância mostrando o efeito do tipo de resíduo cultura (RC), do tipo de solo (S), do tipo de manejo (M), da interação entre RCxS, SxM,RCxM e RCXSxM na decomposição dos resíduos.

\begin{tabular}{cccc}
\hline & \multicolumn{2}{c}{ Decomposição $(\%)$} & \\
& 30 dias & 60 dias & 90 dias \\
\hline $\mathrm{RC}^{\mathrm{a}}$ & $*$ & $*$ & $*$ \\
$\mathrm{~S}^{\mathrm{b}}$ & $*$ & $*$ & $\mathrm{~ns}$ \\
$\mathrm{M}^{\mathrm{c}}$ & $\mathrm{ns}$ & $\mathrm{ns}$ & $*$ \\
$\mathrm{RCXS}$ & $\mathrm{ns}$ & $\mathrm{ns}$ & $\mathrm{ns}$ \\
$\mathrm{SxM}$ & $\mathrm{ns}$ & $\mathrm{ns}$ & $\mathrm{ns}$ \\
$\mathrm{RCxM}$ & $\mathrm{ns}$ & $*$ & $\mathrm{~ns}$ \\
$\mathrm{RCxSxM}$ & $\mathrm{ns}$ & $\mathrm{ns}$ & $\mathrm{ns}$ \\
\hline
\end{tabular}

$\mathrm{RC}^{\mathrm{a}}$ : resíduos culturais de azevém ou nabo forrageiro; $\mathrm{S}^{\mathrm{b}}$ : Argissolo Vermelho Distrófico arênico, em condição de sequeiro ou Planossolo Háplico Eutrófico arênico, em condição de alagamento; $\mathbf{M}^{\mathrm{c}}$ : palha incorporada ao solo ou palha na superfície do solo; $* P<0.05$, ns= não significativo.

\section{CONCLUSÃO}

A decomposição aos 60 dias foi afetada pela interação entre resíduo cultural e manejo, sendo os maiores valores encontrados nos tratamentos com resíduos de nabo forrageiro.

Os resíduos de arroz com maior relação C:N apresentaram os menores valores de decomposição em ambos solos e manejos adotados.

O tipo de solo afetou o processo de decomposição, no Argissolo obteve os maiores valores de decomposição.

\section{REFERÊNCIAS BIBLIOGRÁFICAS}

1. Abiven S, Recous S, Reyes V, Oliver R. Mineralisation of C and N from root, stem and leaf residues in soil and role of their biochemical quality. Biology and Fertility of Soils. 2005 Jun;(42):119-128, doi:10.1007/s00374-005-0006-0.

2. Aita C, Giacomini SJ. Decomposição e liberação de nitrogênio de resíduos culturais de plantas de cobertura de solo solteiras e consorciadas. Revista Brasileira de Ciência do Solo. 2003 Ago;(27):601612, doi:http://dx.doi.org/10.1590/S0100-06832003000400004.

3. Aita C, Giacomini SJ. Plantas de cobertura de solo em sistemas agrícolas. In: Alves, BJR et al. (ed). Manejo de sistemas agrícolas impacto no seqüestro de $\mathrm{C}$ e nas emissões de gases de efeito estufa. Porto Alegre: Genesis, 2006. cap. 3, p. 59-79.

4. Angers DA, Recous S. Decomposition of wheat straw and rye residues as affected by particle size. Plant and Soil. 1997 Feb;(189):197-203, doi:10.1023/A:1004207219678.

5. Carvalho A, Bustamante M, Alcantara F, Resck I, Lemos S. Characterization by solid-state CPMAS 13C NMR spectroscopy of decomposing plant residues in conventional and no-tillage systems in Central Brazil. Soil Tillage Res. 2009 Jan;(102):144-150, doi:10.1016/j.still.2008.08.006.

6. Coppens F, Garnier P, Gryze S, Merckx R, Recous S. Soil moisture, carbon and nitrogen dynamics following incorporation and surface application of labelled crop residues in soil columns. European Journal of Soil Science. 2006 Feb;(57):894-905, doi:10.1111/j.1365-2389.2006.00783.x. 
7. Cotrufo MF, Wallenstein MD, Boot CM, Denef K, Paul E. The Microbial Efficiency-Matrix Stabilization (MEMS) framework integrates plant litter decomposition with soil organic matter stabilization: do labile plant inputs form stable soil organic matter. Global Change Biology. 2013 Apr;(19):988-995, doi:10.1111/gcb.12113.

8. Cotrufo MF, Soong JL, Horton AJ, Campbell EE, Haddix ML, Wall DH, Parton WJ. Formation of soil organic matter via biochemical and physical pathways of litter mass loss. Nature Geoscience. 2015 Sep;(8):766-779, doi:10.1038/ngeo2520.

9. Doneda A. Plantas de cobertura de solo consorciadas e em cultivo solteiro: decomposição e fornecimento de nitrogênio ao milho. Dissertação de Mestrado Universidade Federal de Santa Maria, Centro de Ciências Rurais, Programa de Pós-Graduação em Ciência do Solo, 2010.

10. Finn D, Page K, Catton K, Strounina E, Kienzle M, Robertson F, Armstrong R, Dalal R. Effect of added nitrogen on plant litter decomposition depends on initial soil carbon and nitrogen stoichiometry. Soil Biology \& Biochemistry. 2015 Sep;(91):160-168, doi:10.1016/j.soilbio.2015.09.001.

11. Hadas A, Kautsky L, Goek M, Kara EE. Rates of decomposition of plants residues and available nitrogen in solil, related to residue composition through simulation of carbon and nitrogen turnover. Soil Biology and Biochemestry. 2004 Feb;(36):255-266, doi:10.1016/j.soilbio.2003.09.012.

12. Isaac L, Wood CW, Shanon DA. Decomposition and nitrogen release of prunings from hedgerow species assessed nitrogen release of prunings from hedgerow species assessed for alley croping in Haiti. Agron. 2000 Aug;(92):501-511, doi:10.2134/agronj2000.923501x.

13. Kliemann HJ, Braz AJP B, Silveira PM. Taxas de decomposição de resíduos de espécies de cobertura em Latossolo Vermelho Distroférrico. Pesquisa Agropecuária Tropical. 2006 Abr;(36):21-28.

14. Kramberger B, Gselman a, Janzekovic M, Kaligaric M, Bracko B. Effects of cover crops on soil mineral nitrogen and on the yeald and nitrogen content of maize. European Journal of Agronomy, 2009 May;(31):103-109, doi:10.1016/j.eja.2009.05.006.

15. Lu Y, Watanabe A, Kimura M. Carbon dynamics of rhizodeposites, root and shoot-residues in a rice soil. Soil Biology and Biochemistry. 2003 Sep;(35):1223-1230, doi:org/10.1016/S00380717(03)00184-6.

16. Lupwayi NZ, Clayton GW, O’ Donoban JT, Harker KN, Turkington TK. \& Rice W. A. Decomposition of crop residues under conventional and zero tillage. Canadian Journal of Science. 2004 Jul;(117):3542, doi:10.4141/cjss2012-078.

17. Mazzocini M, Antichi D, Di Bene C, Risaliti R, Petri M, Bonari E. Soil carbono and nitrogen changes after 28 years of no-tillagemanagemente under Mediterranean conditions. European Journal of Agronomy. 2016 Jul;(77):156-175, doi:http://doi.org/10.1016/j.eja.2016.02.011.

18. Neue HU, Gaunt JL, Wang ZP, Beckerheidmann P \& Quijano C. Carbon in tropical wetlands. Geoderma. 1997 Sep;(79):163-185, doi:org/10.1016/S0016-7061(97)00041-4.

19. Olk DC, Dancel MC, Moscoso E, Jimenez RR, Dayrit FM. Accumulation of lignin residues in organic matter fractions of lowland rice soils: a pyrolysis-GC-MS study. Soil Science. 2002 Sep;(167):590606, doi:10.1097/00010694-200209000-00004.

20. Parton WJ, Ojima DS, Schimel DS. Models to evaluate soil organic matter storage and dynamics. In: Carter MR, Stewart BA. (Eds.) Structure and Organic Matter Storage in Soils, Lewis Publ., CRC Press, Boca Raton, FL, 421-448, 1996.

21. Prescott C. Litter decomposition: what controls it and how can we alter it to sequester more carbon in forest soils? Biogeochemistry. 2010 Dec;(101):133-149, doi:10.1007/s10533-010-9439-0.

22. Powlson DS \& Olk DC. Long-term soil organic matter dynamics. In: Kirk GJD \& Olk DC. eds. Carbon and nitrogen dynamics in flooded soils. Los Baños, International Rice Research Institute, 2000, 49-63.

23. Recous S, Robin D, Darwis D \& Mary B. Soil inorganic N Availability: effect on maize residue decomposition. Soil Biology and Biochemistry. 1995 Jul;(27):1529-1538, doi:https://doi.org/10.1016/0038-0717(95)00096-W.

24. Redin, M. Composição bioquímica e decomposição da parte aérea e raízes de culturas comerciais e de plantas de cobertura do solo - Santa Maria, 2010. Dissertação (mestrado) - Universidade Federal de Santa Maria, Centro de Ciências Rurais, Programa de Pós-Graduação em Ciência do Solo, 2010.

25. Robertson GP, Paul EA, Harwood RR. Greenhouse gases in intensive agriculture: contributions of individual gases to the radiative forcing of the atmosphere. Science. 2000 Sep;(289):1922-1925, doi:10.1126/science.289.5486.1922.

26. Silva A, Argenta G, Sangoi L, Strieder ML, Silva A. Estratégias de manejo de coberturas de solo no inverno para cultivo do milho em sucessão no sistema semeadura direta. Ciência Rural. 2006 Jun;(36):1011-1020, doi:http://dx.doi.org/10.1590/50103-84782006000300049.

27. Trinsoutrot I, Recous S, Bentz B. Biochemical quality of crop residues and carbono and nitrogen mineralization kinetics under nonlimiting nitrogen conditions. Soil Science Society of America Journal. 2000 Mar;(64):918-926, doi:10.2136/sssaj2000.643918x. 
28. Valentini CMA. Efluxo de $\mathrm{CO} 2$ do solo de uma área de floresta de transição no noroeste de Mato Grosso. 2004. 81 f. Dissertação (Mestrado em Fisica e meio ambiente) Universidade Federal do Mato Grosso, Cuiabá, 2004. 